\title{
Image and video denoising for distributed optical fibre sensors
}

\author{
Marcelo A. Soto*, Jaime A. Ramírez, Luc Thévenaz \\ EPFL Swiss Federal Institute of Technology, Institute of Electrical Engineering \\ SCI STI LT, Station 11, CH-1015 Lausanne, Switzerland \\ *E-mail: marcelo.soto@epfl.ch
}

\begin{abstract}
A technique based on a multi-dimensional signal processing approach is here described for performance enhancement of distributed optical fibre sensors. In particular, the main features of linear and nonlinear image denoising techniques are described for signal-to-noise ratio enhancement in Brillouin optical time-domain analysers. Experimental results demonstrate the possibility to enhance the performance of distributed Brillouin sensors by more than $13 \mathrm{~dB}$ using a nonlinear image denoising approach, while more than $20 \mathrm{~dB}$ enhancement can be obtained with video denoising.
\end{abstract}

Keywords: Optical fibre sensor, stimulated Brillouin scattering, distributed fibre sensor, image processing

\section{INTRODUCTION}

Distributed optical fibre sensing has experienced a significant evolution during the last decade, becoming a technology that provides unmatched features compared to classical electronic sensors. Exploiting scattering effects along optical fibres (e.g. Raman, Brillouin or Rayleigh scattering), distributed profiles of environmental quantities such as temperature or strain can be measured. Among the different technologies, Brillouin optical time domain analysis (BOTDA) is one of the most performing techniques, allowing for sensing ranges of several tens of kilometres with metric spatial resolution ${ }^{1}$. The most relevant parameter determining the performance of distributed Brillouin sensors (and indeed of all distributed fibre sensing techniques) is the signal-to-noise ratio (SNR) ${ }^{2}$. Unfortunately, the presence of fibre nonlinearities and fibre losses constrain the maximum attainable SNR of the time-domain traces ${ }^{2,3}$, especially at long distances, thus forcing a well-known tradeoff between sensing distance, spatial resolution, measurand uncertainty and acquisition time. Using today's technology, BOTDA sensors can routinely measure over $50 \mathrm{~km}$ with $1 \mathrm{~m}$ spatial resolution and reach a temperature uncertainty below $1 \mathrm{~K}$ in $\sim 1$ minute measurement time. To increase the performance of BOTDA sensors ${ }^{2}$, sophisticated interrogation techniques have been reported in the literature. Significant SNR improvements have been demonstrated using techniques such as distributed optical amplification ${ }^{4}$, pulse coding ${ }^{5}$ or advanced signal processing ${ }^{6}$. For instance, schemes over $>200$ km-long fibre-loops have been reported combining pulse coding and distributed Raman amplification ${ }^{7}$, and more recently using bipolar code sequences ${ }^{8,9}$. On the other hand, combining phase coding and a correlation-domain interrogation ${ }^{10}$, the SNR of a sub-metre resolution Brillouin sensor has been improved, enabling the first demonstration of more than two-million points being resolved in a distributed optical fibre sensor ${ }^{11}$. Unfortunately, most of these achievements have been obtained at the cost of relatively complex and expensive implementations.

This paper describes a technique based on image and video processing to enhance the performance of distributed Brillouin fibre sensors. The method, purely based on a multi-dimensional signal processing approach, can provide a very high SNR enhancement (reaching up to $20 \mathrm{~dB}$ ) with a minor post-processing overhead time and no hardware modifications ${ }^{12-14}$. Although the technique is here described in the context of long-range BOTDA sensing, the same approach can also be applied to boost the SNR of sub-metre resolution systems as well as of other distributed optical fibre sensing technologies, such as distributed Raman or Rayleigh fibre sensors ${ }^{12,15}$.

\section{TWO-DIMENSIONAL IMAGE DENOISING IN BOTDA SENSING}

BOTDA sensors scan the Brillouin gain along a sensing fibre at different pump-probe frequency offsets $\Delta f$. Measured data points are stored in a 2D matrix $g[z, \Delta f]$, which contains the local Brillouin gain spectrum at each fibre position $z$. Image denoising techniques are exploited to reduce the noise present in this measured matrix by processing each positionfrequency pair $\left[z_{i}, \Delta f_{i}\right]$ in $g[z, \Delta f]$ as a noisy pixel of an image ${ }^{12-14}$. This section describes some of the features of linear and nonlinear image filters ${ }^{16}$, providing examples demonstrating the potential of this technique. 


\subsection{Linear image denoising}

Linear image filters make use of 2D neighbourhood (local) operators to reduce noise from noisy pixels in an image ${ }^{16}$. The process can be represented by a two-dimensional convolution: $g_{f}[z, \Delta f]=h[z, \Delta f] * g[z, \Delta f]$, where $h[z, \Delta f]$ is the impulse response of the filter (spatial kernel in the $z$ and $\Delta f$ axes) and $g[z, \Delta f]$ is the original noisy BOTDA data in the $[z, \Delta f]$ space. Due to the linear and time-invariant properties of the filtering process, $2 \mathrm{D}$ linear image denoising can also be expressed in the Fourier domain as: $G_{f}[u, v]=H[u, v] G[u, v]$, where $H[u, v]$ and $G[u, v]$ are the 2D Fourier transform of $h[z, \Delta f]$ and $g[z, \Delta f]$, respectively. This means that there are two equivalent approaches to perform 2D linear image filtering of the BOTDA data: $i$ ) in the spatial domain (i.e. directly in the $[z, \Delta f]$ space) by convolving the data matrix with the 2D filter spatial impulse response, and ii) in the frequency domain by simply multiplying the 2D Fourier transforms of the BOTDA data and filter, and then calculating the 2D inverse Fourier transform of the product. Both approaches are equivalent, existing only differences in the processing time depending on the type of filter.

Some examples of the most common linear image filters are the $2 D$ mean filter (also known as moving averaging filter) and the $2 D$ Gaussian filter. Figure 1 shows the spatial and spectral features of these two filters. Whilst the kernel $h[z, \Delta f]$ of the mean filter (Fig. 1a) is given by a constant value within a well-defined 2D window (i.e. all values within this window are averaged with the same weight), the kernel of the Gaussian filter (Fig. 1c) contains values following a 2D Gaussian shape (i.e. a weighted average of the values $g\left[z_{i}, \Delta f_{i}\right]$ is performed within a 2D window centred in each processed data point $\left.\left[z_{0}, \Delta f_{0}\right]\right)$. Interestingly, Fig. $1 \mathrm{~b}$ points out that the 2D spectral response of the mean filter contains spectral sidelobes, implying that high-frequency noise cannot be fully eliminated from the BOTDA data. Instead, the 2D Gaussian filter can more efficiently attenuate high-frequency components beyond a given well-defined bandwidth, as shown in Fig. 1d. A good trade-off between noise removal and blurring effect can be achieved with the 2D Gaussian filter.
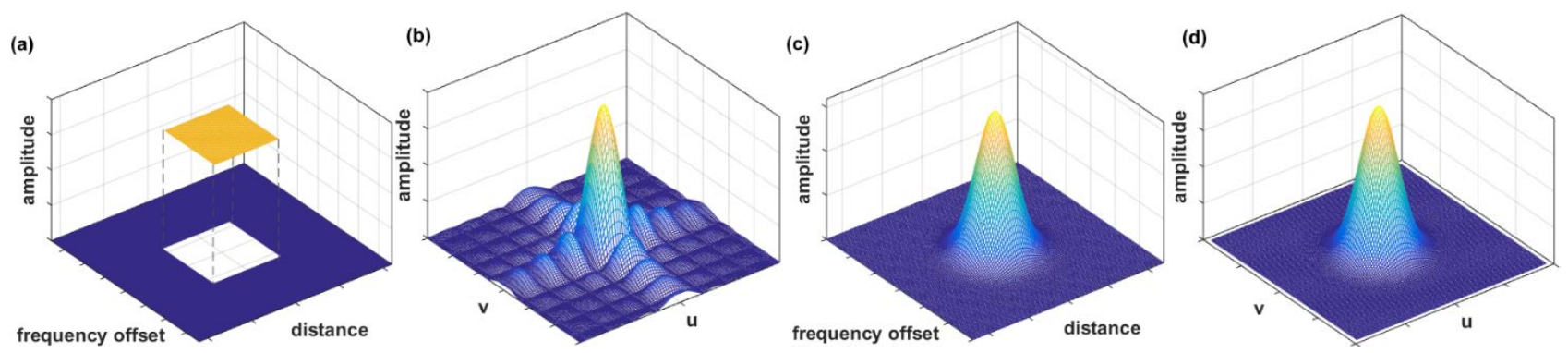

Figure 1. Spatio-spectral response of two linear image filters for BOTDA data denoising. 2D mean filter: (a) Spatial kernel and (b) Fourier transform. 2D Gaussian filter: (c) Spatial kernel and (b) Fourier transform.

It should be highlighted that linear image filters operate as simple 2D low-pass filters, and therefore removing a large amount of noise typically results in blurring and spatial over-smoothing ${ }^{16}$. This can lead to a loss of the high-frequency details contained in the BOTDA data, thus reducing the spatial resolving capability of the sensor. However, although having limited denoising capabilities, 2D linear filters provide a deterministic SNR improvement, which depends mainly on the sampling rate and the target signal bandwidth. For instance, using an isotropic 2D Gaussian kernel with standard deviation of about 2 sampled points, $1 \mathrm{MHz}$ scanning step and sampling interval of $0.5 \mathrm{~m} / \mathrm{pt}$, an SNR improvement of about 7-8 dB has been demonstrated in a 50 km-long BOTDA sensor with $2 \mathrm{~m}$ spatial resolution ${ }^{13}$.

\subsection{Nonlinear image denoising}

Although linear image filters are easy to design and implement, they tend to blur the sharp edges contained in the data, destroying lines and other fine image details. In order to partially overcome the trade-off between spatial smoothing and noise removal, 2D nonlinear image filters can be used. Even though their design and implementation are typically more complex than linear filters, they offer significantly higher SNR enhancement possibilities, while keeping the details of the data. In general, there exist two possible alternatives to implement nonlinear image denoising $\left.{ }^{16-18}: i\right)$ in the spatial domain (i.e. in the $[z, \Delta f]$ space of the BOTDA data), by applying a nonlinear kernel function or nonlinear operator directly to the gain values, and ii) in a transformation domain, by using a nonlinear filtering function in a domain representing the frequency content of the data. After filtering, the denoised data is converted back to the $[z, \Delta f]$ space of the BOTDA data. Examples of the first category, also known as pixel-wise techniques, are the median filter, bilateral filter and non-local means (NLM); whilst transform-based techniques (second category) can be based, for example, on 2D discrete cosine transform or 2D discrete wavelet transform. 
Among these methods, the nonlocal means ${ }^{17}$ takes advantage of the high level of redundancy and similarity contained in the $[z, \Delta f]$ space of the BOTDA data. This approach is ideal for the data measured by a BOTDA sensor, which consist of a spectral resonance peak that is repeatedly measured at each fibre position. The NLM algorithm searches for 2D data patterns with high level of similarity over the data. Denoising is then performed by weighing average, where the weighing factors are determined by the level of similarity between pixels. Figure 2a shows the impact of NLM denoising on the SNR of a $50 \mathrm{~km}$-long BOTDA sensor using 2 time-averaged traces and $2 \mathrm{~m}$ spatial resolution. The figure points out that an SNR of $1.4 \mathrm{~dB}$ at the end of the sensing range can be improved up to $15.2 \mathrm{~dB}$, representing a $13.8 \mathrm{~dB}$ SNR enhancement ${ }^{12}$.

Another example of nonlinear image filtering is the $2 \mathrm{D}$ wavelet denoising, in which the image $g[z, \Delta f]$ is decomposed into sub-images containing different levels of detail ${ }^{16,18}$. Using a nonlinear thresholding function, wavelet shrinkage is applied to the wavelet coefficients obtained by 2D discrete wavelet transform. All wavelet coefficients below a given threshold are associated to noise, and hence set to zero (nonlinear noise elimination function), whilst high-amplitude wavelet coefficients are related to useful information measured by the sensor. By applying 2D inverse wavelet transform to the denoised data, the filtered Brillouin gain is converted back to the $[z, \Delta f]$ space. Figure $2 \mathrm{~b}$ shows the impact of $2 \mathrm{D}$ wavelet denoising (using 5 levels of decomposition and hard thresholding) on the SNR of a $50 \mathrm{~km}$-long BOTDA sensor. Results indicate a 14.2 SNR enhancement, improving the measurement SNR of $1.4 \mathrm{~dB}$ (at $50 \mathrm{~km}$ ) up to $15.6 \mathrm{~dB}^{12}$.

(a)

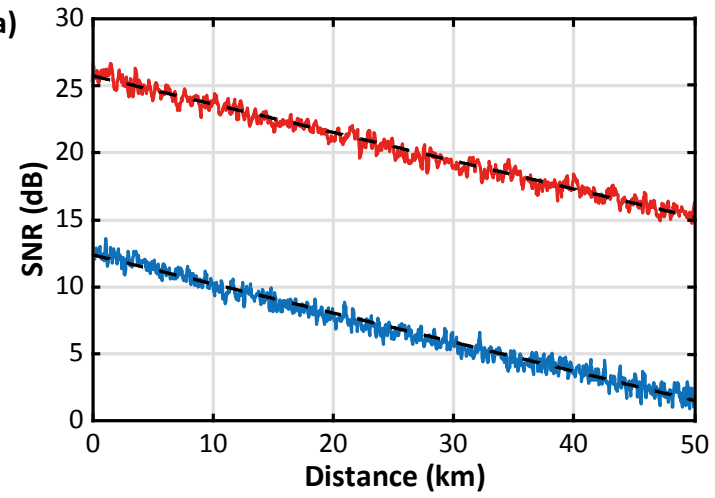

(b)

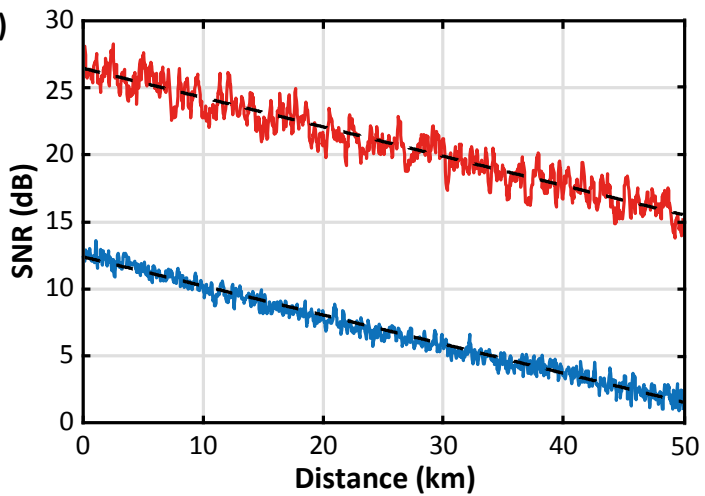

Figure 2. Impact of image denoising on the SNR of a $50 \mathrm{~km}$-long BOTDA sensor. The blue curves represent the SNR vs distance of the raw data and red curves illustrate the SNR obtained after denoising with (a) 2D nonlocal means and (b) 2D wavelet denoising.

It is important to mention that the spatial resolution of $2 \mathrm{~m}$ has been maintained in all the denoised data shown in Fig. 2, which has been verified measuring a 2 m-long hot-spot at the end of the sensing fibre ${ }^{12}$. It should also be noted that the design of nonlinear image filters highly depend on the spatio-spectral features of the data and the noise level, and therefore, the provided SNR enhancement could be different under different scenarios. Optimising a standard BOTDA scheme and the parameters of the NLM filter, sensing over a $200 \mathrm{~km}$-long fibre loop has been recently reported ${ }^{14}$.

\section{THREE-DIMENSIONAL (VIDEO) DENOISING IN BOTDA SENSING}

In order to perform reliable distributed sensing measurements, the acquisition time required to obtain an entire BOTDA measurement (i.e. to perform averaging and full frequency scanning) must be shorter than the temporal evolution of the measurands. This requirement inherently implies that consecutive BOTDA measurements contain some level of correlated information. Processing each BOTDA measurement $g\left[z, \Delta f, t_{k}\right]$ as a frame of a video sequence (being $t_{k}$ the time of the $k$-th acquisition) with a 3D signal processing algorithm can lead to a larger amount of removed noise, when compared to 2D image denoising techniques. This way, video denoising techniques can be used to exploit the redundancy of information found not only in the $[z, \Delta f]$ space of the measurements but also in the temporal dimension ${ }^{12}$. As a result, larger amount of noise can be removed from the measurements when compared to the 2D image denoising approach. Figure 3 indicates that the SNR of a $50 \mathrm{~km}$-long BOTDA

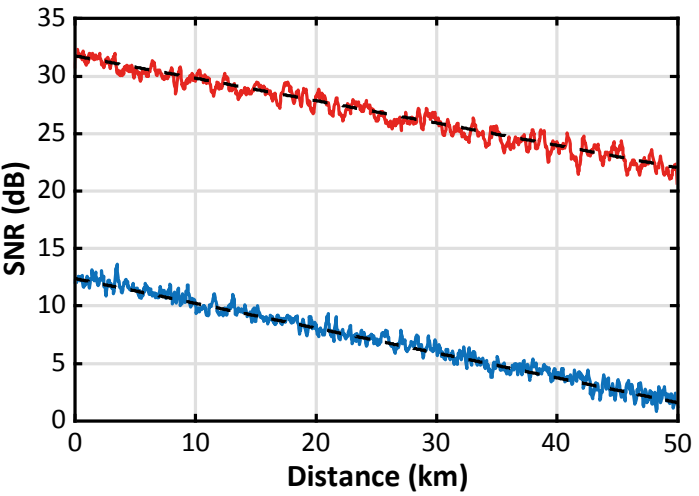

Figure 3. Impact of video denoising on the SNR of a 50 km-long BOTDA sensor. Raw data SNR (blue curve) and SNR obtained with 3D NLM (red curve). 
sensor ( $2 \mathrm{~m}$ spatial resolution, $1.4 \mathrm{~dB}$ original SNR and $42 \mathrm{~s}$ measurement time) can be improved by $20.7 \mathrm{~dB}$ when using a 3D NLM filtering approach (utilising 10 consecutive frames $g\left[z, \Delta f, t_{k}\right]$ for denoising a given frame $g\left[z, \Delta f, t_{0}\right]$ ) A key feature of video denoising is that the processing inherently takes into account the non-stationary characteristics of the data in the temporal domain, and therefore can easily deal with the motion of pixels among different frames. This feature could have interesting applications in dynamic distributed sensing, while for quasi-static sensing it represents a key advantage to minimise delay and over-smoothing effects in the temporal evolution of the measurand ${ }^{12}$.

\section{CONCLUSIONS}

Image and video denoising has demonstrated to be an efficient and powerful tool to remove noise from measurements of distributed optical fibre sensors with a no hardware modifications. This feature makes this multi-dimensional signal processing approach very attractive for a cost-effective industrial development, since the technique can be readily applied to any existing instrument. Although the technique has been here described for long-range BOTDA sensing, the method can be extended (with a suitable adaptation) to other distributed fibre sensing techniques, such as Raman and Rayleigh based fibre sensors, including schemes for sub-metre spatial resolution, distributed dynamic sensing or distributed acoustic sensing, among other potential configurations. The technique can also be combined with other advanced techniques for performance enhancement, such as pulse coding or distributed optical amplification.

\section{REFERENCES}

[1] Motil, A., Bergman, A. and Tur, M., "State of the art of Brillouin fiber-optic distributed sensing," Optics \& Laser Technology 78(A), 81-103 (2016).

[2] Soto, M. A. and Thévenaz, L., "Modeling and evaluating the performance of Brillouin distributed optical fiber sensors,” Opt. Express 21(25), 31347-31366 (2013).

[3] Alem, M., Soto, M. A. and Thévenaz, L., "Analytical model and experimental verification of the critical power for modulation instability in optical fibers,” Opt. Express 23(23), 29514-29532 (2015).

[4] Rodriguez-Barrios, F. et al., "Distributed Brillouin Fiber Sensor Assisted by First-Order Raman Amplification,” J. Lightwave Technol. 28(15), 2162-2172 (2010).

[5] Soto, M. A., Bolognini, G., Di Pasquale, F. and Thévenaz, L., "Simplex-coded BOTDA fiber sensor with $1 \mathrm{~m}$ spatial resolution over a $50 \mathrm{~km}$ range,” Opt. Lett. 35(2), 259-261 (2010).

[6] Farahani, M., Wylie, M., Castillo-Guerra, E. and Colpitts, B., "Reduction in the Number of Averages Required in BOTDA Sensors Using Wavelet Denoising Techniques,” J. Lightwave Technol. 30(8), 1134-1142 (2012).

[7] Soto, M. A. et al., "Extending the real remoteness of long-range Brillouin optical time-domain fiber analyzers," J. Lightwave Technol. 32(1), 152-162 (2014).

[8] Soto, M. A., Le Floch, S. and Thévenaz, L., "Bipolar optical pulse coding for performance enhancement in BOTDA sensors,” Opt. Express 21(14), 16390-16397 (2013).

[9] Yang, Z., Soto, M. A. and Thévenaz, L., "Increasing robustness of bipolar pulse coding in Brillouin distributed fiber sensors,” Opt. Express 24(1), 586-597 (2016).

[10]Zadok, A., Antman, Y., Primerov, N., Denisov, A., Sancho, J. and Thévenaz, L., "Random-access distributed fiber sensing,” Laser \& Photon. Rev. 6, L1-L5 (2012).

[11] Denisov, A., Soto, M. A. and Thévenaz, L., "Going beyond 1000000 resolved points in a Brillouin distributed fiber sensor: theoretical analysis and experimental demonstration,” Light: Science \& Applications 5, e16074 (2016).

[12] Soto, M. A., Ramírez, J. A. and Thévenaz, L., "Intensifying the response of distributed optical fibre sensors using 2D and 3D image restoration," Nat. Commun. 7, 10870 (2016).

[13] Soto, M. A., Ramírez, J. A. and Thévenaz, L., "Intensifying Brillouin distributed fibre sensors using image processing,” Proc. SPIE 9634, 24th International Conference on Optical Fibre Sensors, 96342D (2015).

[14] Soto, M. A., Ramírez, J. A. and Thévenaz, L., "200 km Fiber-Loop Conventional Brillouin Distributed Sensor with 2m Spatial Resolution,” 6th Asia Pacific Optical Sensors (APOS) Conference, paper Th3A.4 (2016).

[15] Soto, M. A., Ramírez, J. A. and Thévenaz, L., "Reaching millikelvin resolution in Raman distributed temperature sensing using image processing,” Proc. SPIE 9916, $6^{\text {th }}$ European Workshop on Optical Fibre Sensors, 99162A (2016).

[16] Szeliski, R., “Computer Vision: Algorithms and Applications”. Springer Science \& Business Media, 2010.

[17] Buades, A., Coll, B. and Morel, J. M., "A review of image denoising methods, with a new one,” Multiscale Model. Simul. 4(2), 490-530 (2005).

[18] Mallat, S. G., “A theory for multiresolution signal decomposition: the wavelet representation,” IEEE Trans. Pattern Anal. Mach. Intell. 11, 674-693 (1989). 